\title{
Surgical Management of the Kidney Transplant Recipient
}

\author{
Cheguevara Afaneh, Meredith J. Aull, Anthony Watkins, \\ Sandip Kapur and Jim Kim
}

Additional information is available at the end of the chapter

http://dx.doi.org/10.5772/54145

\section{Introduction}

The early postoperative course and management can have a significant impact on the longterm success of a kidney transplant recipient. Several factors affect long-term outcomes including the occurrence of delayed graft function (DGF), episodes of acute rejection (AR), surgical complications, and overwhelming infections, especially sepsis [1-3]. Certain medications, including calcineurin inhibitors $(\mathrm{CNI})$, also have potential for nephrotoxic effects, which can later lead to transplant glomerulopathy [4]. Furthermore, recipient characteristics, such as sensitization status, and donor characteristics, such as donation after cardiac death (DCD) donors and expanded criteria donors (ECD) can all affect long-term outcomes [5]. Although basic postoperative surgical principles are applied, there are certain parameters that need to be closely monitored, especially as it pertains to fluid management, blood pressure control, and immunologic status. Early detection of graft dysfunction is paramount in determining reversibility from both medical and surgical complications. Recognizing the technical limitations during surgery can also help prevent potentially devastating mechanical complications. Thus, appropriate initial management and mitigation of various risk factors is extremely important in the long-term success of the kidney transplant patient.

\section{Surgical procedure}

Technical variations exist for kidney transplant, such as in the retroperitoneal exposure or implantation of the ureter, but the basic surgical procedure will be described in this section. The right iliac fossa has traditionally been described as the initial choice for implantation, but previous operations, quality of vessels, or other recipient factors may make the left side 
more approachable. In the case of polycystic kidney disease, where the native kidneys need to be removed, a midline incision is made.

\subsection{Backtable kidney preparation}

The kidney is kept cold in an ice slush bath during the preparation, which involves dissecting the renal artery and vein from the surrounding tissue. The ureter is identified and retracted away so as to avoid injury when removing extraneous fat and tissue from the kidney and hilum. Attention must be directed to protecting the blood supply to the ureter by avoiding the so-called "golden triangle" at the inferior pole of the kidney. Multiple renal arteries may also require repair and a Carrel patch may or may not be preserved depending on the degree of aortic plaque seen. In the case of a right kidney, the renal vein may require lengthening, which can be accomplished by utilizing the attached IVC.

\subsection{Surgical exposure}

An oblique curvilinear incision is made in the right or left lower quadrant of the abdomen, extending from near the pubic symphysis to above the anterior superior iliac spine of the iliac crest. Muscle layers can be directly divided, lateral to the rectus sheath, or split along the fibers of the external and internal obliques and transversalis. The peritoneum is identified and retracted superiorly and medially to expose the retroperitoneum. Selfretaining retractors can be placed to facilitate subsequent exposure of the psoas and iliac vessels.

\subsection{Operative procedure}

The common or external iliac artery and vein are identified and dissected. Lymphatics that course along the length of the vessels need to be meticulously tied or cauterized to prevent occurrence of lymphoceles. Vascular flow is controlled proximally and distally with vascular clamps and the kidney is brought into the surgical field. A venotomy is first made in the recipient iliac vein and an end-to-side anastomosis with the renal vein is created with 5-0 or 6-0 monofilament non-absorbable suture. Similarly, an arteriotomy is then made and the arterial anastomosis is completed in an end-to-side fashion. The clamps are released sequentially, with the vein before the artery, and the kidney is perfused. Once hemostasis is attained, the urinary tract is reconstructed. The bladder, which should be irrigated with antibiotic solution prior to start of the procedure, can be filled by way of a three-way Foley catheter or instilled with the antibiotic solution at the start of the operation. The kidney is positioned in the retroperitoneum and an area on the bladder is identified for implantation of the ureter. The layers of the bladder are carefully dissected and a cystostomy is created. The transplant ureter is cut to length and the anastomosis is done with or without a stent using monofilament absorbable suture. One technique described for ureteroneocystostomy is the Lich-Gregoir technique, which involves an anastomosis between the ureteral and bladder mucosa with a myotomy closure over the ureter. The abdominal wall is then reapproximated and closed in layers. 


\section{Early postoperative course}

\subsection{Renal and fluid management}

The initial management of the kidney transplant recipient involves proper fluid management, focusing on volume status and electrolyte balance. Assessing volume status is multifaceted and includes monitoring urine output, central venous pressure, heart rate, and blood pressure. Attention to daily weights and total input-output tabulations can help dictate fluid management, especially regarding the use of diuretics postoperatively. A decrease in urine volume can result from hypovolemia, obstruction, acute tubular necrosis (ATN), urinary leak, or in the most severe case, vascular thrombosis.

Recipients of living donor kidney transplants have brisk urine output immediately or within minutes of implantation. Fluids may need to be replaced adequately to avoid a negative fluid balance within the first 24 hours. This can potentially compromise blood flow to the new kidney. Deceased donor allografts, in comparison, not make significant urine amounts initially. Fluid and furosemide challenges should be considered; however, if there has been little to no response after several attempts, then fluids should be administered judiciously in consideration of overall volume status from a respiratory, cardiovascular, and renal standpoint. The goal of fluid resuscitation in the early postoperative period focuses on maintaining good perfusion to the transplanted allograft.

Changes in urine output should be assessed in an objective and systematic manner. First, the foley should be assessed for patency and flushed, as patients may have mild hematuria leading to clot formation. If hypovolemia is suspected, then a fluid challenge with crystalloid or albumin should be administered [6]. Failure to respond to a fluid challenge and increases in serum creatinine should prompt assessment of the graft with Duplex ultrasonography (DUS), which can be used to assess perfusion to the allograft, rule out hydronephrosis and evaluate perinephric fluid collections. Significant, but less dramatic decreases in urine output should raise clinical suspicion for renal artery or vein thrombosis, which would warrant surgical re-exploration if caught in a timely fashion. Patients with little to no response to fluid challenges should also be administered a furosemide challenge. Patients who fail to respond to fluid or furosemide without any structural or vascular abnormalities on DUS may have ATN, which can be confirmed with a biopsy.

\subsection{Cardiovascular and pulmonary assessment}

Assessment and maintenance of adequate blood pressure control is imperative to the success of the kidney transplant. Because the transplanted kidney is an end-organ, it is susceptible to injury during episodes of hypotension, which can lead to ATN. Careful attention to the choice of induction therapy being administered, such as rabbit antithymocyte globulin (rATG), is important as the side effect profile includes fever, dyspnea, respiratory distress, and hypotension [7]. If other causes of respiratory distress or hypotension have been excluded, the rate of RATG administration may need to be slowed or even stopped temporarily or permanently. Basiliximab, an interleukin-2 receptor antagonist 
(IL2RA), has fewer side effects and can be administered as an alternative under different immunosuppression protocols.

The use of calcium channel blockers has been shown to be beneficial in kidney transplantation. Intra-arterial administration of calcium channel blockers, such as verapamil, improves renal blood flow as well as augments immunosuppression [8]. Postoperative administration of oral calcium channel blockers has also been implemented, as there is evidence that the incidence of DGF is decreased [9]. In a large systematic review and metaanalysis of randomized controlled trials, the use of calcium channel blockers has been shown to decrease the risk of graft loss and improved post-transplant glomerular filtration rates (GFR) [10]. Furthermore, in the early transplant period, the use of calcium channel blockers have been shown to be superior to angiotensin-converting enzyme inhibitors with regards to avoiding nephrotoxicity, improving GFR, improving hemoglobin levels, minimizing the incidence of hyperkalemia, and minimizing proteinuria post-transplant [10].

Respiratory complications can lead to poor outcomes. As previously stated, the use of rATG may lead to respiratory distress as capillary leak can occur. Acute respiratory failure posttransplant can compromise allograft outcomes [11,12]. The leading cause of respiratory failure is typically bacterial pneumonia [12]. Patients with prolonged intensive care hospitalizations are at risk of invasive fungal and opportunistic infections, especially in the setting of intense perioperative immunosuppression. These infections have been linked to increased mortality [13]. Appropriate chemoprophylaxis with trimethoprimsulfamethoxazole for Pneumocystis jiroveci pneumonia may be beneficial and potentially mitigate the infectious risks [14].

\section{Assessment of graft function}

\subsection{Early graft dysfunction}

Early complications leading to graft dysfunction can be separated into two categories: medical or surgical. Marginal donors, including ECD and DCD allografts, have the highest rate of medical and surgical complications [15,16]. Various medical and surgical complications resulting in early graft dysfunction are listed in Table 1. The following sections will discuss the most common culprits in each category. Hypovolemia has been discussed in a previous section.

\subsection{Primary non-function}

Primary non-function (PNF), defined as the lack of adequate allograft function by the third month post transplant, has a reported incidence between 1-8\% [17-19]. Typically, patients at risk of PNF include highly sensitized patients and those on renal replacement therapy for a prolonged duration of time prior to transplantation. Acute rejection and surgical complications are the most common causes of PNF [19]. The use of histidine-tryptophanketoglutarate (HTK) solution has also been implicated as a cause of PNF in deceased donor renal transplants [17]. A recent study suggests that a mean arterial blood pressure less than 
or equal to $80 \mathrm{~mm} \mathrm{Hg}$ approximately 3 months before kidney transplantation is a risk factor for PNF [18]. Certain donor factors, such as prolonged cold ischemia time, have also been associated with PNF [20]. Patients with PNF have poorer overall patient survival, compared to patients with immediate graft function, probably as a result of returning to renal replacement therapy at an earlier time.

\begin{tabular}{|c|c|}
\hline \multicolumn{2}{|c|}{ Common Causes of Early Graft Dysfunction } \\
Medical & Surgical \\
\hline Delayed graft function & Hemorrhage \\
Hypovolemia & Urinary obstruction \\
Acute rejection & Vascular thrombosis \\
Drug-induced nephrotoxicity & Hematuria \\
Infection & Arterial stenosis \\
Disease recurrence & Extrinsic obstruction \\
\hline
\end{tabular}

Table 1. Common causes of early graft dysfunction stratified by medical or surgical causes.

\subsection{Immunologic events}

\subsubsection{Hyperacute rejection}

Hyperacute rejection occurs in the setting of $\mathrm{ABO}$ incompatible transplants or in the setting of a positive lymphocytotoxic crossmatch, where the incidence is approximately $85 \%$. Some antibodies may have lower binding affinity to receptors or fail to bind any complement. Once this process has occurred, treatment involves immediate removal of the allograft. Some protocols utilizing plasmapheresis have been used with modest results [21], but graft survival remains poor. The emergence of kidney-paired donation and chains has circumvented the need for transplantation across ABO blood groups [22]. Renal scan typically demonstrates no perfusion and pathology reveals microvascular thrombosis, thus necessitating a transplant nephrectomy. With the advent of modern immunologic testing prior to transplant, hyperacute rejection remains a rare occurrence.

\subsubsection{Accelerated vascular rejection}

Accelerated vascular rejection is an early aggressive form of acute rejection that may occur in sensitized recipients with a high panel-reactive antibody or patients with a previous transplant, despite a negative $\mathrm{T}$ cell crossmatch. This type of rejection occurs as early as postoperative day 2 or can be as late as postoperative day 5. Anti-rejection treatment modalities generally fail to be effective in these patients. Histology reveals fibrin deposition and endothelitis. These patients are typically treated similar to antibody-mediated rejection episodes with plasmapheresis, intravenous immunoglobulin, and/or antibody-depleting agents [23]. 


\subsubsection{Acute rejection}

Acute rejection can occur at any time in the early post-transplant period. The most common time point, however, is 5 to 7 days post-transplant. Acute cellular rejection remains the most common type of rejection episode. The incidence of acute rejection is highest in the first 6 months post-transplant, with overall acute rejection rates between $10-20 \%$. The immunologic profiles of the donor and recipient as well as the use of different immunosuppression protocols are important in stratifying acute rejection risk. Signs and symptoms of acute rejection include fever, elevated serum creatinine, increasing weight, and graft tenderness. Transplant biopsy remains the gold standard for diagnosis. Histological changes in acute rejection include tubulitis and interstitial infiltrates, with or without arteritis [24].

Treatment of acute cellular rejection depends on the severity. First-line therapy includes a steroid pulse. In more severe cases of acute rejection, an antibody-depleting agent, such as rATG should be administered in addition to a steroid pulse. CNI levels should be monitored closely in the setting of acute rejection, as nephrotoxicity is more common. Moreover, in the setting of antibody-depleting therapy, patients should be monitored closely for infectious complications [25]. Graft survival is negatively impacted by episodes of acute rejection [26].

\section{Technical complications}

\subsection{Vascular}

\subsubsection{Renal artery thrombosis}

Renal artery thrombosis is a rare event. Technical issues, such as arterial kinking or intimal dissection, are the usual culprits. The majority of these events occur in the first few days following transplantation. Sudden cessation of urine production should raise the suspicion for vascular compromise. Risk factors for this devastating condition include the use of pediatric donors less than or equal to $15 \mathrm{~kg}$ without an aortic patch as well as kidneys from donors less than 5 years of age $[27,28]$. However, use of pediatric donors less than $10 \mathrm{~kg}$ has also been successfully reported without an increased rate of arterial thrombosis [29]. The diagnosis must be made promptly, as the allograft can only tolerate 30-60 minutes of warm ischemia before the allograft has irreversible injury. Even in ideal situations, recovery from ischemic injury may still be impossible. A high-index of suspicion is necessary to ensure prompt and adequate treatment of this condition with emergent re-operation. In cases where the recipient had previously made urine at baseline, the diagnosis becomes even more difficult. The diagnosis is made with DUS, which demonstrate lack of color flow (Figure 1).

When multiple renal arteries are present on the donor allograft, reconstruction may be necessary during implantation. Most authors have reported no difference in vascular complications or graft survival [30]. However, thrombosis of some minor branches supplying superior or inferior poles can lead to partial infarction of the renal allograft. Patients may present with elevated serum creatinine or hypertension. Subsequent angiogram will demonstrate a wedge perfusion defect of the allograft. Patients may then present with urine leak if caliceal infarction is present. In such circumstances, the patient may benefit from 
nephrostomy tube placement for complete urinary decompression as well as percutaneous drain placement in the event of urinoma development.

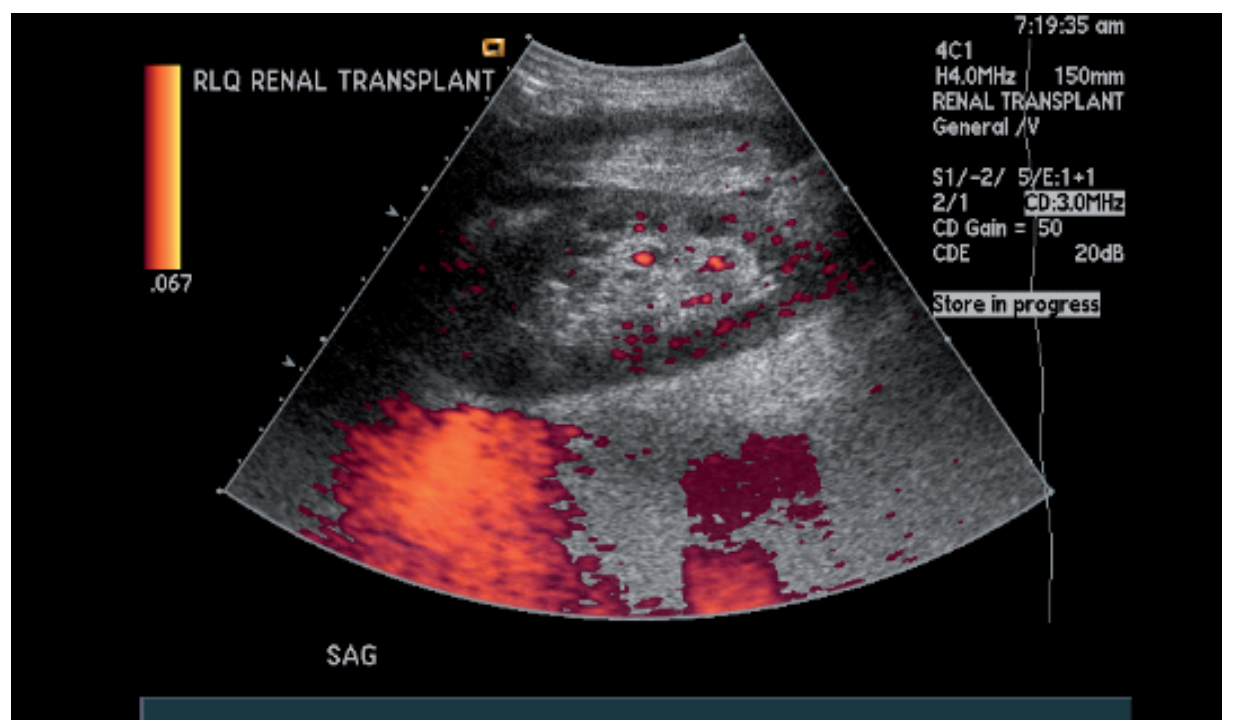

Figure 1. Doppler ultrasound demonstrates global hypoperfusion of the transplanted kidney consistent with renal artery thrombosis on postoperative day 1 .

\subsubsection{Renal vein thrombosis}

Renal vein thrombosis is also an uncommon, but potentially devastating, complication. The usual causes include injuries to the donor renal vein that was narrowed after repair of an injury or twisting of the vein on the renal pedicle. Previously, the use of right-sided living donor renal allografts was associated with an increased risk of renal vein thrombosis due to the short length. However, we have not seen this in our large series of right-sided donors, even with modern procurement techniques, such as laparoendoscopic single-site surgery [31,32]. Patients present with gross hematuria, decreased urine output and engorgement of the graft due to venous outflow obstruction. DUS will show parvus tardus and reversal of diastolic flow in the renal artery. Immediate repair is necessary if there is to be any chance of salvaging the allograft.

\subsubsection{Hemorrhage and hematoma}

Most postoperative hematomas are small and insignificant. They are usually incidentally found on a post-transplant DUS. Larger and more clinically significant hematomas may occur in the setting of antiplatelet therapy or anticoagulation for patients with risk factors for venothromboembolic events (Figure 2). Patients with clinically significant hematomas will present with swelling from the incision, pain over the graft, and an acute drop in hemoglobin values. The hematoma will continue to increase in size until it ultimately impinges on the vascular pedicle of the allograft, which can lead to thrombosis or 
hydronephrosis. Postoperatively, patients may show signs of bruising in dependent regions of the wound, flank and groin.

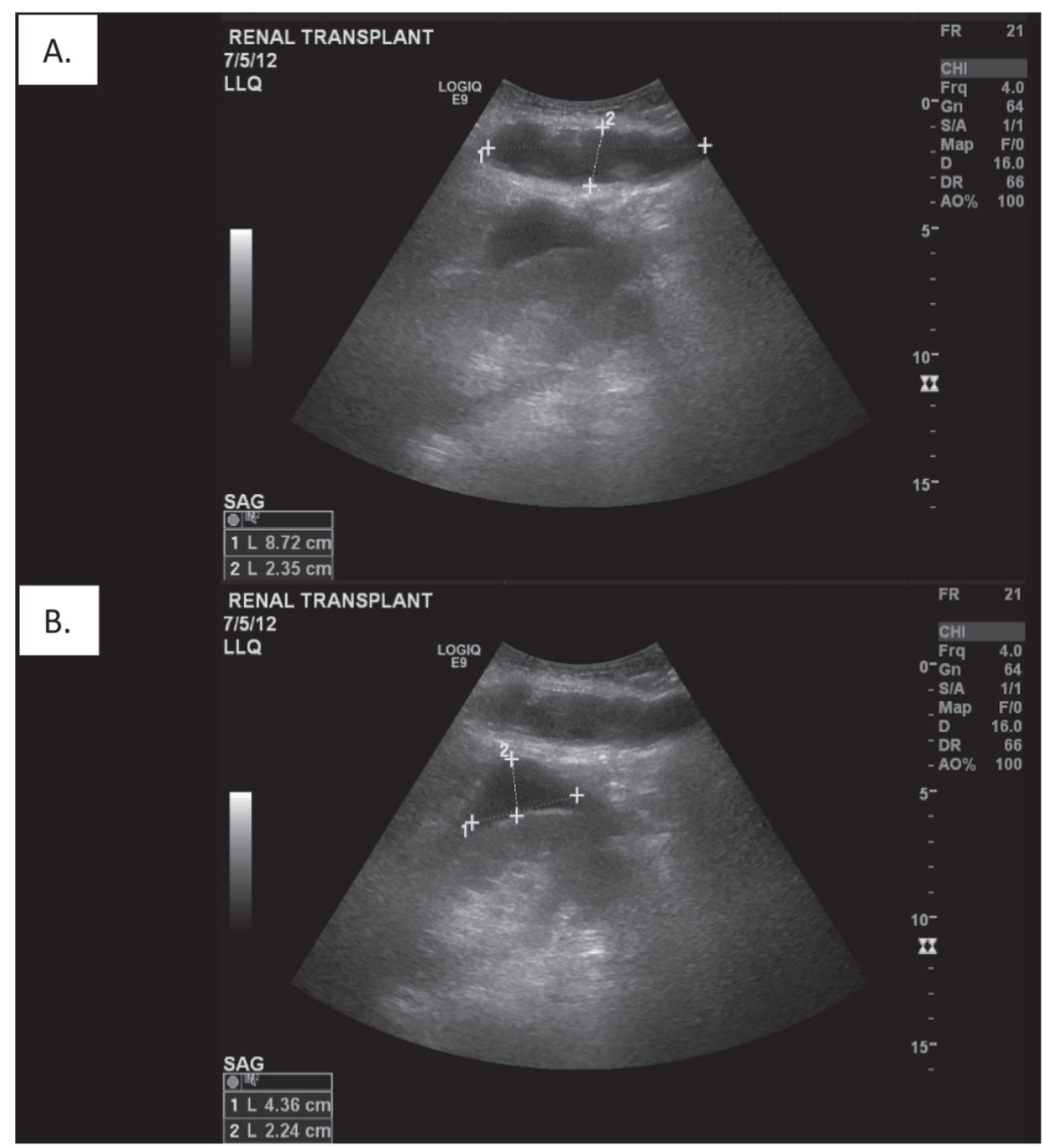

Figure 2. Panel A demonstrates an early postoperative hematoma (as outlined by plus signs) above the fascia in a transplant patient on systemic anticoagulation. Panel B illustrates a perinephric hematoma (as outlined by plus signs) in the same patient.

Patients requiring postoperative anticoagulation for prophylaxis of a vascular thrombotic event are at greatest risk of developing a hematoma [33,34]. The reported risk of a postoperative bleed on heparin requiring surgical exploration has been reported as high as $60 \%$ in patients on anticoagulation. Moreover, patients with a history of lupus and lupus anticoagulant are especially sensitive to heparin anticoagulation, leading to an increased risk of postoperative hemorrhage [35]. Percutaneous drainage of large hematomas is insufficient and operative exploration and evacuation should be undertaken to avoid vascular thrombosis and to remove clot as a potential nidus of infection. 


\subsubsection{Renal artery stenosis}

Transplant renal artery stenosis is a late complication of kidney transplantation. The diagnosis is usually made with DUS, which demonstrates parvus tardus waveforms as well as elevated resistive indices. An MRA is necessary to confirm the diagnosis. Treatment includes interventional procedures involving balloon angioplasty and potentially stenting or surgical repair with cadaveric graft. This topic is discussed in detail in another chapter of this textbook.

\subsection{Urinary}

\subsubsection{Ureteral obstruction}

Pelvicaliceal dilation seen on DUS implies obstruction in the urinary flow. Placement of a foley catheter and examination of its patency can provide relief if the cause is from an enlarged prostate or dysfunctional bladder. Failure of the foley catheter to relieve obstruction necessitates immediate decompression via placement of a percutaneous nephrostomy tube. An antegrade pyelogram can be performed to visualize where the obstruction occurs (Figure 3). A decrease in the serum creatinine following decompression confirms the diagnosis. After 1-2 days of allowing the postoperative edema to subside, a nephrogram is performed to evaluate whether ureteral obstruction or stenosis remains. Early strictures usually require surgical repair, where as late strictures are more amenable to less invasive procedures, such as stent placement and angioplasty.

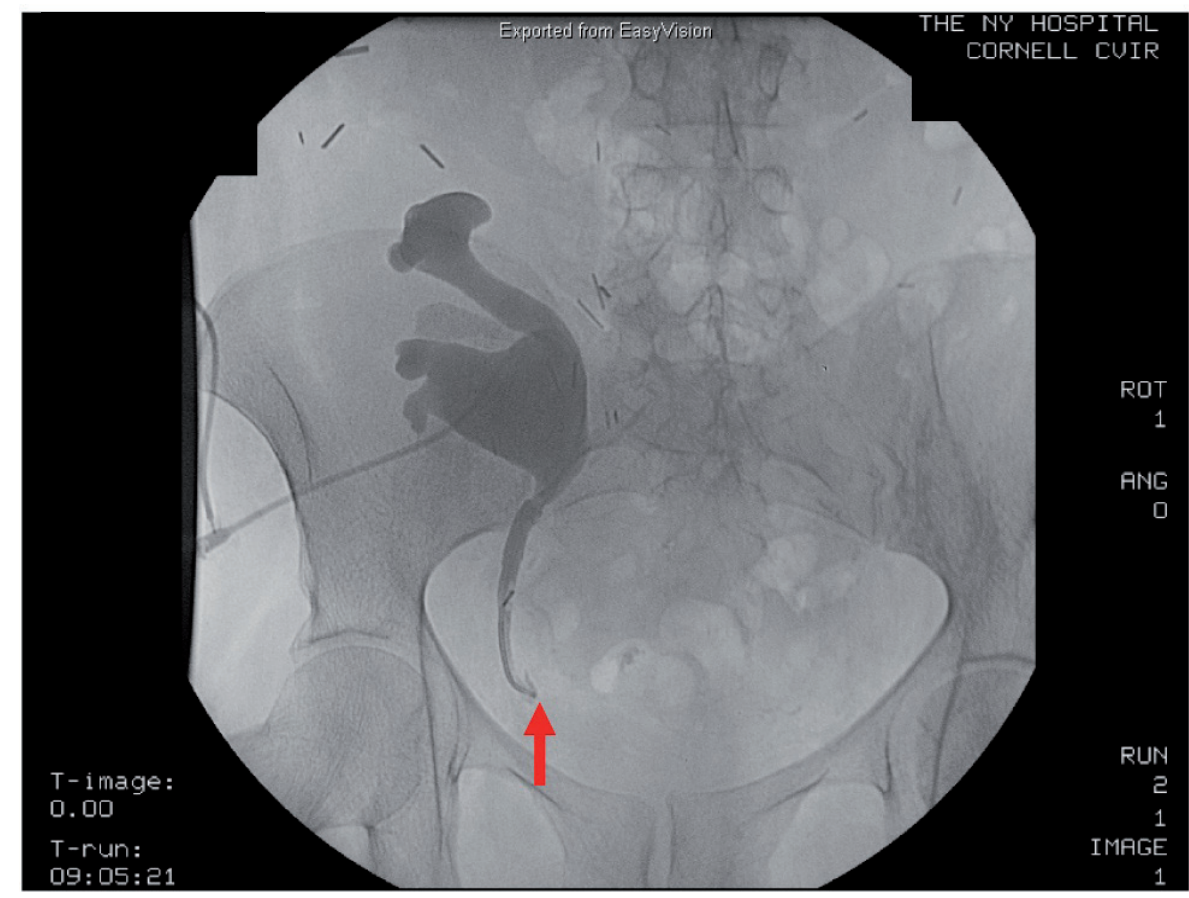

Figure 3. An antegrade pyelogram is shown demonstrating complete obstruction (red arrow) at the ureteroneocystostomy anastomosis of the transplanted kidney in the right lower quadrant. 
Ureteral strictures in the early postoperative period are related to technical issues. Patients with ureteral stents will not present with obstruction in the early postoperative period. Rather they may present with this problem several weeks after stent removal. This complication is usually the result of a twist in the ureter or narrowing at the anastomosis due to distal ureteral ischemia [36]. Short segment strictures $(<2 \mathrm{~cm})$ are usually amenable to percutaneous dilatation whereas longer strictures $(>2 \mathrm{~cm})$ and those involving the proximal or mid-ureter typically require surgical revision. The treatment options include excising the stricture and creating a new ureteroneocystostomy, if sufficient length is available on the transplant ureter. A psoas hitch or Boari flap can be performed to bring the bladder closer to the kidney to assist in this approach. Otherwise, a ureteroureterostomy using the ipsilateral native ureter or ureteropyelostomy may be required. A 6 French double-J stent can be left in place for 4 to 6 weeks. In cases where the ipsilateral native ureter is unavailable, the contralateral ureter can also be used. Graft survival is not significantly affected in patients undergoing correction or revision of urologic complications in the first year post-transplant [37].

\subsubsection{Urine leak}

Urine leak usually occurs in the first month after transplant and is due to a disruption in the ureteral-bladder anastomosis. Caliceal infarction from a partial thrombosis of the renal allograft can also present as a urine leak. The presenting symptoms include abdominal pain, increasing serum creatinine level and a decrease in urine output. Typically, a DUS or renal scan shows a fluid collection in the retroperitoneal space (Figure 4). Sampling of the fluid and analysis for creatinine can confirm the diagnosis of a urinoma.

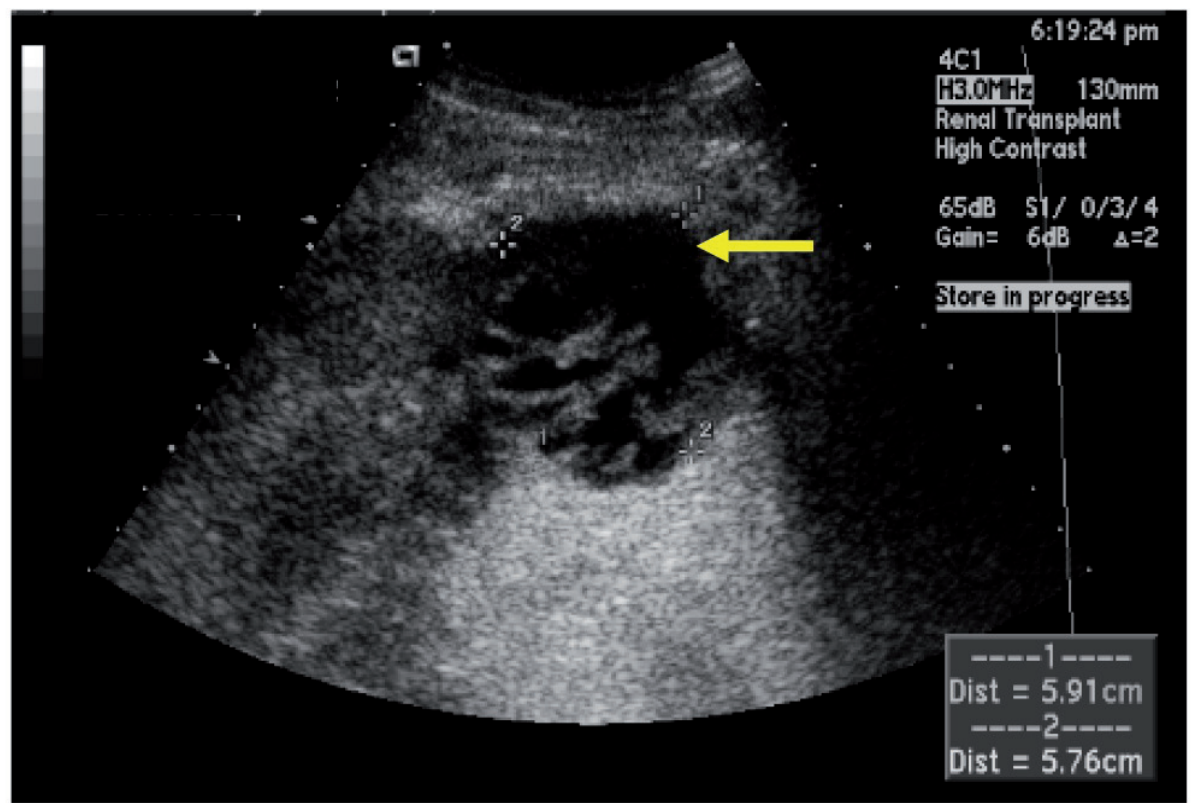

Figure 4. Urinoma (yellow arrow) located at the inferior pole of the transplanted kidney as a result of a urine leak. 
Causes of urine leak include technical issues related to creation of the ureteroneocystostomy or ischemic necrosis of the distal ureter. Urgent surgical management of this condition is mandatory to decrease the risk of wound infection and improve potential for recovery of the allograft.

\subsection{Lymphocele}

A lymphocele is a collection of lymphatic fluid that develops in the postoperative field in a nonepithelialized cavity. These collections typically form around the divided lymphatics surrounding the recipient iliac vasculature. Most lymphoceles are asymptomatic and do not require any intervention; however, lymphoceles can cause compressive symptoms from mass effect on surrounding tissue and structures, or can become infected. Most lymphoceles occur between 2 weeks and 6 months post-transplant [38]. The peak incidence is about 6 weeks post-transplant. The source of the lymphocele is from disrupted lymphatic channels surrounding the iliac vasculature during implantation. Lymphocele formation can be prevented by meticulous dissection and ligation of all lymphatic trunks with a nonabsorbable suture. Some have even suggested that lymphocele formation can be avoided by anastomosing to the common iliac vasculature were less lymphatic tissue is present [39]. The use of mTOR inhibitors has been strongly and positively correlated with the development of lymphoceles in kidney transplantation [40].

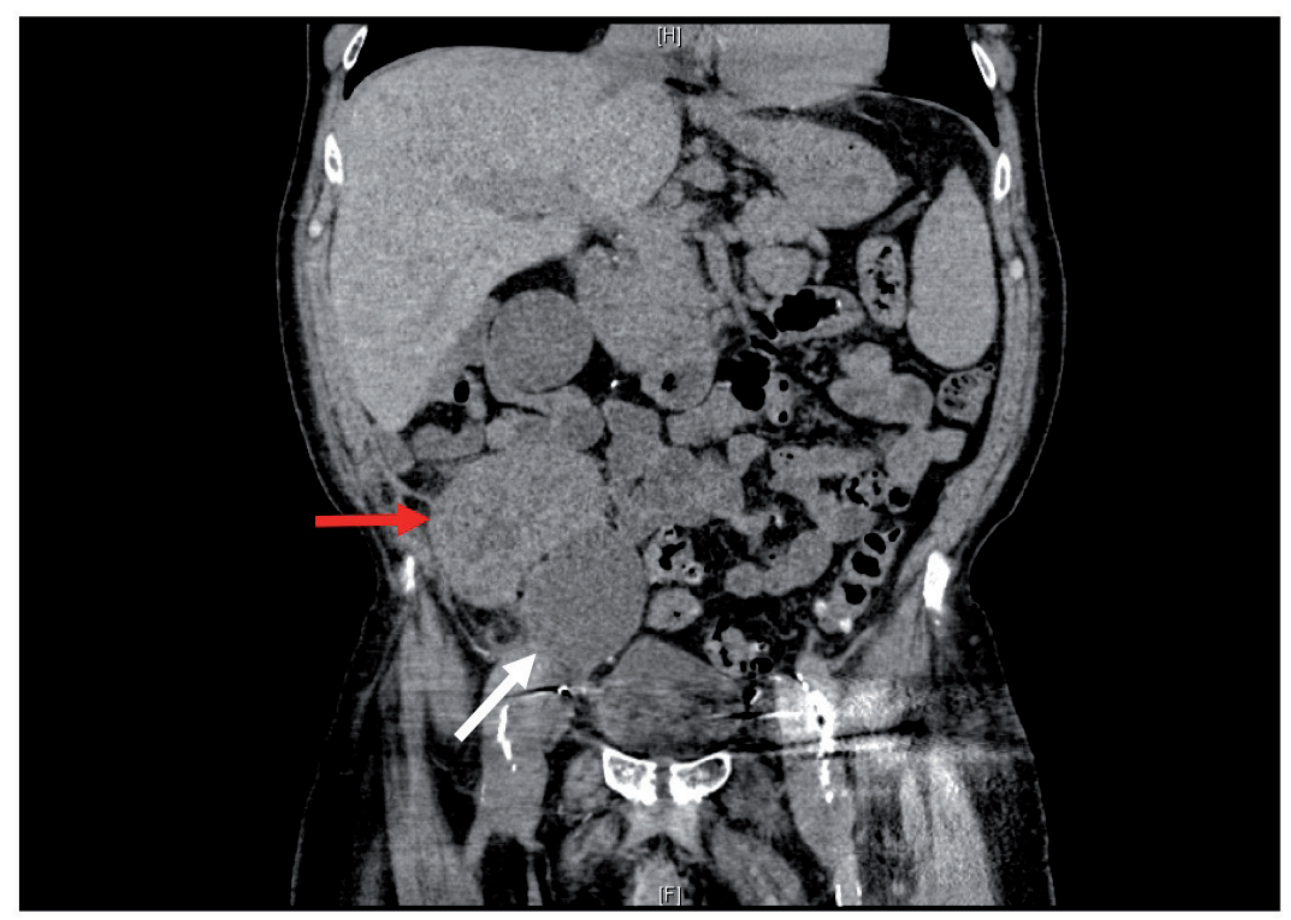

Figure 5. A lymphocele (white arrow) can be seen on CT scan compressing the right lower quadrant transplanted kidney (red arrow) causing hydronephrosis. 
Lymphoceles are suggested by DUS or CT scan findings demonstrating a large fluid periallograft collection (Figure 5). The most common location is adjacent to the bladder and multiple collections may be present. Sending aspirated fluid for cytological and biochemical analysis for lymphocytes can confirm the diagnosis. Small, asymptomatic collections should be left alone, as spontaneous resolution is common. Larger collections and those that are causing obstructive symptoms can be initially managed by placement of a percutaneous drain. Recurrence is common and several aspirations may be necessary. Each aspiration carries a theoretical risk of infection. Sclerotherapy with povidone-iodine can be effective [41]. A possible complication of povidone-iodine is acute renal failure and this should be monitored during and after treatment [42]. Operative drainage of lymphoceles provides definitive therapy. The goal for drainage is to allow communication with the peritoneal cavity where it can be reabsorbed. This can be done via an open or the preferred laparoscopic approach [38]. The lymphocele is unroofed or fenestrated with a $5 \mathrm{~cm}$ opening to allow direct drainage into the peritoneal cavity.

\section{Medical complications}

\subsection{Delayed graft function}

Delayed graft function (DGF) is the most common cause of early graft dysfunction and is defined as the need for dialysis within the first week after transplant. DGF has been reported to affect approximately $20-25 \%$ of all deceased donor transplant recipients [43]. Recipients of marginal donors, including ECD and DCD, have rates of DGF as high as $70 \%$ in some studies $[15,44]$. The use of pulsatile hypothermic machine perfusion has been shown in studies to reduce that risk, though the overall rate still remains higher than SCD allografts [44]. Various donor risk factors for DGF include age, cause of death, and ischemia reperfusion injury [45].

DGF is a well-documented risk factor for poor graft survival. In a 2011 study analyzing 40 years of deceased donor renal transplant recipients, the occurrence of DGF and acute rejection were the most significant predictors of renal allograft survival rates [26]. As previously discussed, marginal donors have the highest rates of DGF, while the rate of DGF in living donors is relatively uncommon. Additionally, patients undergoing retransplantation have higher rates of DGF than first time recipients of renal allografts. ECD allograft recipients may have a higher rate of acute rejection, which may be related to the higher rate of DGF in these allografts [46]. Prolonged cold ischemia has been a welldocumented cause of DGF; however, recent data suggests that cold ischemia-induced DGF may play a limited role in long-term outcomes [47].

The use of DUS and biopsy can differentiate DGF from other causes of early graft dysfunction, such as acute rejection or surgical complications. A transplant biopsy is usually necessary to differentiate between other causal factors, such as acute rejection or recurrent disease. Radiographic studies in the proper clinical context can also aid in the diagnosis of DGF. Typically, transplants with DGF will demonstrate good renal perfusion and good parenchymal uptake of radionucleotide tracer with little or no renal excretion. Primary nonfunction should be considered in the differential at this time point. 


\subsection{Drug-induced nephrotoxicity}

Calcineurin inhibitors (CNIs) have become the cornerstone of immunosuppression regimens. Early administration post-transplant has helped mitigate the risk of acute rejection in kidney transplantation. Most institutions have implemented the use of polyclonal antibody-depleting or monoclonal antibody induction therapy in conjunction with introduction of CNIs as early as postoperative day 1 or 2 , as we do in our institution. CNIs can have a nephrotoxic effect on the allograft by decreasing renal blood flow in the afferent arteriole, leading to tubular injury [48]. Variable oral absorption of CNIs, especially cyclosporine, in the early postoperative period can lead to either overdosing or underdosing, causing either nephrotoxicity or acute rejection, respectively.

Differentiating calcineurin inhibitor toxicity from other causes of graft dysfunction is difficult based on clinical context. Percutaneous transplant biopsy can be used to diagnose other causes of graft dysfunction. Histologic findings are non-specific with tubular injury and tubular vacuolization being the most common early findings. Hyaline deposition and fibrosis can be found with chronic injury. Obtaining daily calcineurin inhibitor levels can prevent supratherapeutic dosing, predisposing patients to the nephrotoxic effects. Avoiding nephrotoxicity is important, as chronic nephrotoxicity has been shown to correlate with chronic transplant nephropathy, ultimately affecting long-term allograft survival [49].

\subsection{Infectious complications}

Infectious complications in the early postoperative period are typically related to the operation. The most common postoperative infections include surgical site infections, urinary tract infections, bacteremia from central venous catheters, and pneumonia [50]. Careful and meticulous surgical technique and attention to detail can help prevent most early infectious complications, such as surgical site infections, central venous catheter sepsis, and urinary tract infections from foley catheter placement. Encouraging early ambulation and the use of incentive spirometry decreases the incidence of atelectasis and the risk of postoperative pneumonia. Removing foley catheters and stents once they no longer serve an appreciable purpose can help prevent and eliminate urinary tract infections. Central venous catheters should be removed as early as possible to decrease the incidence of line sepsis.

Typically, opportunistic infections are relatively uncommon in the early postoperative period (<30 days). Cytomegalovirus (CMV) infection occurs, especially in seronegative recipients receiving allografts from seropositive donors. Institution of CMV prophylaxis with oral valganciclovir or high-dose oral acyclovir for a minimum of 3 months has significantly reduced the incidence and severity of CMV infection [51]. Once antiviral prophylaxis has been halted, CMV infection may still occur. Appropriate prophylaxis with trimethoprim/sulfamethoxazole has almost entirely eliminated early Pneumocystis carinii pneumonia. Prophylactic antifungal agents, such as nystatin or clotrimazole troches, have also been instituted to decrease the risk of oral Candida infections in the early postoperative period. 
Prompt diagnosis and treatment of infectious complications leads to better outcomes. Aggressive treatment with intravenous antibacterial, antiviral or antifungal agents for severe infections improve outcomes. Infected intra-abdominal collections should be drained. Minimizing external instrumentation should be part of the fundamental strategy in preventing infectious complications.

\subsection{Disease recurrence}

The majority of causes of end-stage renal disease (ESRD) do not recur early in the posttransplant period. Disease processes such as diabetes mellitus and hypertension can cause recurrence of ESRD if poorly controlled over a long period of time. Two causes of ESRD, however, can recur immediately post-transplant: focal segmental glomerulosclerosis (FSGS) and thrombotic microangiopathy (TMA).

FSGS is a form of glomerulonephritis that can recur immediately post-transplant. Although the exact mechanism is unclear, a serum factor causes glomerular injury and early massive proteinuria [52,53]. The development of nephrotic range proteinuria can be suggestive of disease recurrence in a patient with a pretransplant diagnosis of FSGS. The diagnosis can be verified with electron microscopy demonstrating effacement of foot processes. Current treatment strategies include increasing calcineurin inhibitor dosing, steroids, and plasmapheresis, with the latter modality being the most effective [52]. If patients fail to respond over several weeks, then further treatments are not likely to be effective.

Thrombotic microangiopathy can also recur relatively quickly post-transplant. Causes of TMA include recurrent disease, endothelial damage from calcineurin inhibitor use, hypercoagulable disorders, or antibody-mediated rejection episodes [54]. Clinical manifestations include hemolysis and decreases in hemoglobin, platelet count, and haptoglobin. Additionally, microangiopathy will be present on peripheral blood smears. Patients will have increases in lactate dehydrogenase and serum creatinine, signifying allograft dysfunction. Transplant biopsy will show fibrin clot in arterioles. Treatment includes removing inciting factors, such as calcineurin inhibitors, as well as plasmapheresis [55]. Eculizumab, a humanized monoclonal antibody to complement component 5 (C5) to mediate complement-mediated injury, has emerged as a possible treatment option for TMA [56]. Blocking complement activation, especially the last step of the complement cascade, has important implications in TMA. Treatment of TMA posttransplant has been successfully reported [57], but still carries a poor prognosis.

\section{Summary}

Early management of the kidney transplant recipient is crucial for optimizing outcomes and avoiding early graft loss, or even death. Given the surgical and medical complexity of these patients, attention to detail and prompt diagnosis of complications is critical to achieving excellent outcomes. Clinical scenarios need to be recognized where early operative intervention is necessary to save the graft. However, whenever possible, prevention of complications provides the best outcomes. 


\section{Author details}

Cheguevara Afaneh, Meredith J. Aull, Anthony Watkins, Sandip Kapur and Jim Kim Department of Surgery, Division of Transplant Surgery, New York-Presbyterian Hospital- Weill Cornell Medical College, New York, NY, USA

\section{References}

[1] Almond, P.S.; Matas, A.; Gillingham, K., et al. (1993). Risk factors for chronic rejection in renal allograft recipients. Transplantation, Vol.55, No.4, (April 1993), pp. 752-6, ISSN 0041-1337.

[2] Aultman, D.F.; Sawaya, D.E.; Zibari, G.B., et al. (1999). Are all successful renal transplants really successful? Am J Kidney Dis, Vol.34, No.1, (July 1999), pp. 61-4, ISSN 0272-6386.

[3] Troppmann, C.; Gillingham, K.J.; Benedetti, E., et al. (1995). Delayed graft function, acute rejection, and outcome after cadaver renal transplantation: a multivariate analysis. Transplantation, Vol.59, No.7, (April 1995), pp. 962-8, ISSN 0041-1337.

[4] Nankivell, B.J.; Borrows, R.J.; Fung, C.L., et al. (2003). The natural history of chronic allograft nephropathy. N Engl J Med, Vol.349, No.24, (December 2003), pp. 2326-33, ISSN 0028-4793.

[5] Matsuoka, L.; Shah, T.; Aswad, S., et al. (2006). Pulsatile perfusion reduces the incidence of delayed graft function in expanded criteria donor kidney transplantation. Am J Transplant, Vol.6, No.6, (June 2006), pp. 1473-8, ISSN 1600-6135.

[6] Dawidson, I.J.; Sandor, Z.F.; Coorpender, L., et al. (1992). Intraoperative albumin administration affects the outcome of cadaver renal transplantation. Transplantation, Vol.53, No.4., (April 1992), pp. 774-82, ISSN 0041-1337.

[7] Buchler, M.; Hurault de Ligny, B.; Madec, C., et al. (2003). Induction therapy by antithymocyte globulin (rabbit) in renal transplantation: a 1-yr follow-up of safety and efficacy. Clin Transplant, Vol.17, No.6, (December 2003), pp. 539-45, ISSN 0902-0063.

[8] Dawidson, I.; Rooth, P.; Fry, W.R., et al. (1989). Prevention of acute cyclosporineinduced renal blood flow inhibition and improved immunosuppression with verapamil. Transplantation, Vol.48, No.4, (October 1989), pp. 575-80, ISSN 0041-1337.

[9] Dawidson, I.; Rooth, P.; Alway, C., et al. (1990). Verapamil prevents posttransplant delayed function and cyclosporine A nephrotoxicity. Transplant Proc, Vol.22, No.4, (August 1990), pp. 1379-80, ISSN 0041-1345.

[10] Cross, N.B.; Webster, A.C.; Masson, P.; O'connell, P.J.; Craig, J.C. (2009). Antihypertensives for kidney transplant recipients: systematic review and meta-analysis of randomized controlled trials. Transplantation, Vol.88, No.1, (July 2009), pp. 7-18, ISSN 0041-1337.

[11] Candan, S.; Pirat, A.; Varol, G.; Torgay, A.; Zeynelgolu, P.; Arslan, G. (2006). Respiratory problems in renal transplant recipients admitted to intensive care during long-term followup. Transplant Proc, Vol.38, No.5, (June 2006), pp. 1354-6, ISSN 0041-1345.

[12] Canet, E.; Osman, D.; Lambert, J., et al. (2011). Acute respiratory failure in kidney transplant recipients: a multicenter study. Crit Care, Vol.15, No.2, (March 2011), R91, ISSN 1364-8535.

[13] Sharifipour, F.; Resaeetalab, F.; Naghibi, M. (2009). Pulmonary fungal infection in kidney transplant recipients: an 8-year study. Transplant Proc, Vol.41, No.5, (June 2009), pp. 1654-6, ISSN 0041-1345. 
[14] Green, H.; Paul, M.; Vidal, L.; Leibovici, L. (2007). Prophylaxis for pneumocystis pneumonia (PCP) in non-HIV immunocompromised patients. Cochrane Database Syst Rev, No.3, (July 2007), pp. CD005590, ISSN 1469-493X.

[15] Barba, J.; Zudaire, J.J.; Robles, J.E.; Rosell, D.; Berian, J.M.; Pascual, I. (2012). Complications of kidney transplantation with grafts from expanded criteria donors. World J Urol, (Jan 2012), Epub ahead of print, ISSN 1433-8726.

[16] Ledinh, H.; Weekers, L.; Bonvoisin, C., et al. (2012). Results of kidney transplantation from controlled donors after cardio-circulatory death: a single center experience. Transplant Int, Vol.25, No.2, (February 2012), pp. 201-9, ISSN 0934-0874.

[17] Stevens, R.B.; Skorupa, J.Y.; Rigley, T.H., et al. (2009). Increased primary non-function in transplanted deceased-donor kidneys flushed with histidine-tryptophan-ketoglutarate solution. Am J Transplant, Vol.9, No.5, (May 2009), pp. 1055-62, ISSN 1600-6135.

[18] Webber, A.; Hambleton, J.; Chami, A., et al. (2012). Mean arterial blood pressure while awaiting kidney transplantation is associated with the risk of primary nonfunction. Transplantation, Vol.93, No.1, (January 2012), pp. 54-60, ISSN 0041-1337.

[19] Woo, Y.M.; Jardine, A.G.; Clark, A.F., et al. (1999).Early graft function and patient survival following cadaveric renal transplantation. Kidney Int, Vol.55, No.2, (February 1999), pp. 692-9, ISSN 0085-2538.

[20] van der Vliet JA, Warlé MC, Cheung CLS, Teerenstra S, Hoitsma AJ. Influence of prolonged cold ischemia in renal transplantation. Clin Transplant 2011: 25: E612-E616

[21] Squifflet, J.P.; De Meyer, M.; Malaise, J.; Latinne, D.; Pirson, Y.; Alexandre, G.P. (2004). Lessons learned from ABO-incompatible living donor kidney transplantation: 20 years later. Exp Clin Transplant, Vol.2, No.1, (June 2004), pp. 208-13, ISSN 1304-0855.

[22] Leeser, D.B.; Aull, M.J.; Afaneh, C., et al. (2012). Living donor kidney paired donation transplantation: experience as a founding member center of the National Kidney Registry. Clin Transplant, Vol.26, No.3, (May/June 2012), pp. E213-22, ISSN 0902-0063.

[23] Jordan, S.C.; Vo, A.A.; Tyan, D.; Nast, C.C.; Toyoda, M. (2005). Current approaches to treatment of antibody-mediated rejection. Pediatr Transplant, Vol.9, No.3, (June 2005), pp. 408-15, ISSN 1399-3046.

[24] Racusen, L.C.; Solez, K.; Colvin, R.B., et al. (1999). The Banff 97 working classification of renal allograft pathology. Kidney Int, Vol.55, No.2, (February 1999), pp. 713-23, ISSN 0085-2538.

[25] Jamil, B.; Nicholls, K.; Becker, G.J.; Walker, R.G. (1999). Impact of acute rejection therapy on infections and malignancies in renal transplant recipients. Transplantation, Vol.68, No.10, (November 1999), pp. 1597-603, ISSN 0041-1337.

[26] Serur, D.; Saal, S.; Wang, J, et al. (2011). Deceased-donor kidney transplantation: improvement in long-term survival. Nephrol Dial Transplant, Vol.26, No.1, (January 2011), pp. 317-24, ISSN 0931-0509.

[27] Mohanka, R.; Basu, A.; Shapiro, A.; Kayler, L.K. (2008). Single versus en bloc kidney transplantation from pediatric donors less than or equal to $15 \mathrm{~kg}$. Transplantation, Vol.86, No.2, (July 2008), pp. 264-8, ISSN 0041-1337.

[28] Singh, A.; Stablein, D.; Tejani, A. (1997). Risk factors for vascular thrombosis in pediatric renal transplantation: a special report of the North American Pediatric Renal Transplant Cooperative Study. Transplantation, Vol.63, No.9, (May 1997), pp. 1263-7, ISSN 0041-1337. 
[29] Balachandran, V.P.; Aull, M.J.; Goris, M.; Figueiro, J.; Leeser, D.B.; Kapur, S. (2010). Successful transplantation of single kidneys from pediatric donors weighing less than or equal to $10 \mathrm{~kg}$ in standard weight adult recipients. Transplantation, Vol.90, No.5, (September 2010), pp. 518-22, ISSN 0041-1337.

[30] Benedetti, E.; Troppmann, C.; Gillingham, K., et al. (1995). Short- and long-term outcomes of kidney transplants with multiple renal arteries. Ann Surg, Vol.221, No.4, (April 1995), pp. 406-14, ISSN 1528-1140.

[31] Afaneh, C.; Ramasamy, R.; Leeser, D.B., et al. (2011a). Is right-sided laparoendoscopic single-site donor nephrectomy feasible? Urology, Vol.77, No.6, (June 2011), pp. 13651369, ISSN 0090-4295.

[32] Afaneh, C.; Aull, M.J.; Schubl, S., et al. (2011b). The evolution of laparoscopic right donor nephrectomy: Progression to single site surgery. J Transplant Technol Res, Vol.1, Issue.2, No.103, (November 2011), pp. 1-7, ISSN 2161-0991.

[33] Friedman, G.S.; Meier-Kriesche, H.U.; Kaplan, B., et al. Hypercoagulable states in renal transplant candidates: impact of anticoagulation upon incidence of renal allograft thrombosis. Transplantation, Vol.72, No.6, (September 2011), pp. 1073-8, ISSN 0041-1337.

[34] Kusyk, T.; Verran, D.; Stewart, G., et al. (2005). Increased risk of hemorrhagic complications in renal allograft recipients receiving systemic heparin early posttransplantation. Transplant Proc, Vol.73, No.2, (March 2005), pp. 1026-8, ISSN 0041-1345.

[35] Mathis, A.S. \& Shah, N.K. (2004). Exaggerated response to heparin in post-operative renal transplant recipient with lupus anticoagulant undergoing plasmapheresis. Transplantation, Vol.77, No.6, (March 2004), pp. 957-8, ISSN 0041-1337.

[36] Ghasemian, S.M.; Guleria, A.S.; Khawand, N.Y.; Light, J.A. (1996). Diagnosis and management of urologic complications of renal transplantation. Clin Transplant, Vol.10, No.2, (April 1996), pp. 218-23, ISSN 0902-0063.

[37] van Roijen, J.H.; Kirkels, W.J.; Zietse, R.; Roodnat, J.I.; Weimar, W.; Ijzermans, J.N. (2001). Long-term graft survival after urological complications of 695 kidney transplantations. J Urol, Vol.165, No.6 pt 1, (June 2001), pp. 1884-7, ISSN 0022-5347.

[38] Bailey, S.H.; Mone, M.C.; Holman, J.M.; Nelson, E.W. (2003). Laparoscopic treatment of post renal transplant lymphoceles. Surg Endosc, Vol.17, No.12, (December 2003), pp. 1896-9, ISSN 1432-2218.

[39] Sansalone, C.V.; Aseni, P.; Minetti, E., et al. (2000). Is lymphocele in renal transplantation an avoidable complication? Am J Surg, Vol.179, No.3, (March 2000), pp. 182-5, ISSN 0002-9610.

[40] Pengel, L.H.; Liu, L.Q.; Morris, P.J. (2011). Do wound complications or lymphoceles occur more often in solid organ transplant recipients on mTOR inhibitors? A systematic review of randomized controlled trials. Transplant Int, Vol.24, No.12, (December 2011), pp. 1216-30, ISSN 0934-0874.

[41] Montalvo, B.M.; Yrizarry, J.M.; Casillas, V.J., et al. (1996). Percutaneous sclerotherapy of lymphoceles related to renal transplantation. J Vasc Interv Radiol, Vol.7, No.1, (JanuaryFebruary 1996), pp. 117-23, ISSN 1051-0443.

[42] Manfro, R.C.; Comerlato, L.; Berdichevski, R.H., et al. (2002). Nephrotoxic acute renal failure in a renal transplant patient with recurrent lymphocele treated with povidone-iodine irrigation. Am J Kidney Dis, Vol.40, No.3, (September 2002), pp. 655-7, ISSN 0272-6386. 
[43] Cecka, J.M. (2003). The UNOS Renal Transplant Registry. In Cecka JM. Terasaki PI (eds): Clinical Transplants 2002. Los Angeles, UCLA Immunogenetics Center, 2003, pp. 1-20, ISSN 0890-9016.

[44] Jochmans, I.; Moers, C.; Smits, J.M., et al. (2010). Machine perfusion versus cold storage for the preservation of kidneys donated after cardiac death: a multicenter, randomized, controlled trial. Ann Surg, Vol.252, No.5, (November 2010), pp. 756-64, ISSN 1528-1140.

[45] Land, W. (1998). Postischemic reperfusion injury and kidney transplantation. Transplant Proc, Vol.30, No.8, (December 1998), pp. 4210-3, ISSN 0041-1345.

[46] Cecka, J.M. (2005). The UNOS Renal Transplant Registry. In Cecka JM. Terasaki PI (eds): Clinical Transplants 2004. Los Angeles, UCLA Immunogenetics Center, 2003, pp. 1-16, ISSN 0890-9016.

[47] Kayler, L.K.; Srinivas, T.R.; Schold, J.D. (2011). Influence of CIT-induced DGF on kidney transplant outcomes. Am J Transplant, Vol.11, No.12, (December 2011), pp. 2657-64, ISSN 1600-6135.

[48] Perico, N.; Ruggenenti, P.; Gaspari, F., et al. (1992). Daily renal hypoperfusion induced by cyclosporine in patients with renal transplantation. Transplantation, Vol.54, No.1, (July 1992), pp. 56-60, ISSN 0041-1337.

[49] Solez, K., Vincenti, F.; Filo, R.S. (1998). Histopathologic findings from 2-year protocol biopsies from a U.S. multicenter kidney transplant trial comparing tacrolimus versus cyclosporine: a report of the FK506 Kidney Transplant Study Group. Transplantation, Vol.66, No.12, (December 1998), pp. 1736-40, ISSN 0041-1337.

[50] Rubin, R.H. Infectious diseases in transplantation/pre- and post-transplantation. In Norman DJ, Suki, WN (eds): Primer on Transplantation. Thorofare, NJ, American Society of Transplant Physicians, 1998, pp. 141-152.

[51] Paya, C.; Humar, A.; Dominguez, E., et al. (2004). Efficacy and safety of valganciclovir vs. oral ganciclovir for prevention of cytomegalovirus disease in solid organ transplant recipients. Am J Transplant, Vol.4, No.4, (April 2004), pp. 611-20, ISSN 1600-6135.

[52] Artero, M.L.; Sharma, R.; Savin, V.J.; Vincentin, F. (1994). Plasmapheresis reduces proteinuria and serum capacity to injure glomeruli in patients with recurrent focal glomerulosclerosis. Am J Kidney Dis, Vol.23, No.4, (April 1994), pp. 574-81, ISSN 0272-6386.

[53] Savin, V.J.; Sharma, R.; Sharma, M., et al. (1996). Circulating factor associated with increased glomerular permeability to albumin in recurrent focal segmental glomerulosclerosis. $N$ Engl J Med, Vol.334, No.14, (April 1996), pp. 878-83, ISSN 0028-4793.

[54] Chiurchiu, C.; Ruggenenti, P.; Remuzzi, G. (2002). Thrombotic microangiopathy in renal transplantation. Ann Transplant, Vol.7, No.1, (2002), pp. 28-33, ISSN 1425-9524.

[55] Kaplan, A.A. (2001). Therapeutic apheresis for renal disease. Ther Apher, Vol.5, No.2, (April 2001), pp.134-41, ISSN 1744-9979.

[56] Fehr, T. \& Gaspert, A. (2012). Antibody-mediated kidney allograft rejection: therapeutic options and their experimental rationale. Transpl Int, Vol.25, No.6, (June 2012), pp. 62332, ISSN 0934-0874.

[57] Wilson, C.H.; Brown, A.L.; White, S.A.; Goodship, T.H.; Sheerin, N.S.; Manas, D.M. (2011). Successful treatment of de novo posttransplant thrombotic microangiopathy with eculizumab. Transplantation, Vol.92, No.8, (October 2011), pp. e42-3, ISSN 0041-1337. 